\title{
THE EVOLUTION OF STELLAR CORONAE: INITIAL RESULTS FROM A ROSAT PSPC OBSERVATION OF IC 2391
}

\author{
Brian M. Patten and Theodore Simon ${ }^{1}$ \\ Institute for Astronomy, University of Hawaii, 2680 Woodlawn Drive, Honolulu, HI 96822 \\ Received 1993 May 24; accepted 1993 July 14
}

\begin{abstract}
A $23 \mathrm{ks}$ ROSAT PSPC image of the young star cluster, IC 2391, reveals 76 soft X-ray sources with $L_{x}(0.2-2.0 \mathrm{keV}) \geq 2 \times 10^{28} \mathrm{ergs} \mathrm{s}^{-1}$ in the direction of the cluster center. Nineteen of these sources are associated with known cluster members. We find that X-ray emission from the IC 2391 B stars deviates widely from the $L_{x} / L_{b o l}=10^{-7}$ relation based on Einstein observations of $O$ and early $B$ stars. Instead, we observe a wide range in $L_{\mathbf{x}}$ with an order of magnitude spread at any given mass and no apparent dependence on spectral type. A comparison of the spread of $L_{x}$ as a function of $B-V$ for low-mass stars between IC 2391 and the much older Hyades cluster shows that despite the factor of $\sim 10$ difference in their ages, these two clusters exhibit very similar dispersions in levels of stellar activity. We conclude that the low-mass stars in IC 2391 have arrived on the ZAMS with a wide range of coronal activity levels, from very strong to very weak, and that existing empirical activity-age scaling laws therefore cannot be valid.
\end{abstract}

Subject headings: open clusters and associations: individual (IC 2391) - stars: coronae - stars: early-type -stars: late-type - X-rays: stars

\section{INTRODUCTION}

Over the last two decades a number of dynamo activity-age relations have been proposed for late-type stars, based on the strength of various chromospheric/transition region emission features (Skumanich 1972; Barry, Cromwell, \& Hege 1987; Simon, Herbig, \& Boesgaard 1985). Recent discussion in the literature, however, questions whether unique activity-age relations really exist for main sequence stars, or whether stars instead arrive on the ZAMS with a wide range of activity levels and then subsequently follow their own decay laws (Simon $1990,1992)$. With the advent of the ROSAT X-ray mission, we now have an opportunity to conduct a more complete census of coronal activity levels in young star clusters and to explore this question in considerable detail.

IC 2391 is a young southern cluster, which contains about three dozen known members ranging in spectral type from $B$ to $M$. It has an age, determined through fitting of model isochrones, of 30-50 Myr (Stauffer et al. 1989), making it younger than both of its better known northern counterparts, $\alpha$ Per and the Pleiades. Most of the low-mass stars in IC 2391 have just arrived on the ZAMS, making this cluster a perfect laboratory for testing activity-age relations. To that end, we have obtained a deep X-ray image of IC 2391 with the ROSAT Position Sensitive Proportional Counter (PSPC), and in this Letter we present the initial results of our survey of coronal activity in this cluster.

\section{OBSERVATIONS AND ANALYSIS}

IC 2391 was observed with the PSPC in 18 separate time intervals over the period 1992 May 19 to June 25, for a total accepted time of $22,926 \mathrm{~s}$. Our observation (see Fig. 1 [Pl. 5]) was positioned southeast of the brightest cluster member, $o$ Vel, a B3/5 V star, at the nominal center of the distribution of low-mass cluster members identified by Stauffer et al. (1989). Although the membership of IC 2391 has often been termed

' $\operatorname{ROSAT}$ Guesı Observer. "sparse," a large number of X-ray sources is seen in this image. Many of these sources are likely associated with previously unidentified cluster members. The number of sources in our image is similar to that seen in a PSPC observation of the Pleiades (Caillaut, Gagné, \& Stauffer 1992).

The data were analyzed using the Post-Reduction Off-line Software (PROS) running under the IRAF software package. $X$-ray source positions were determined using the PROS DETECT package with a variety of detection cell sizes. Each detected source was manually checked to verify its existence in the PSPC image. A few bright sources at large off-axis angles that could not be identified by the PROS DETECT routines were identified and centroided by hand. In total, 76 sources with a signal-to-noise ratio $>3.0$ were identified in the image. The errors associated with our photometry are dominated by photon statistics. A comparison of the sources detected with PROS to the source list generated by the standard pipeline processing (SASS) shows generally good agreement in the $X$-ray source positions, although the SASS processing did fail to identify several bright sources in the crowded central portion of the image.

An initial survey of this image was made by assembling a list of known cluster members from the published literature (Hogg 1960; Buscombe 1965; Perry \& Hill 1969; Perry \& Bond 1969; Levato \& Malaroda 1984; Levato \& Garcia 1984; Stauffer et al. 1989; Levato et al. 1988). We selected 33 members within the PSPC field of view and compared their positions on the sky to those of the $X$-ray sources. To aid in identification, the positions of the X-ray sources were overplotted on a digitally scanned ESO plate containing the cluster. Nineteen of the 33 member stars were found to have $X$-ray counterparts in the PSPC image. The average offset between the $X$-ray source position and the stellar position was $12^{\prime \prime}$, while the largest was 22 ". For the remaining stars, the offset to the nearest X-ray source was greater than $60^{\prime \prime}$, which is twice the positional accuracy of the PSPC. Figure 2 shows a sky plot of the cluster members and the $\mathrm{X}$-ray source positions. 


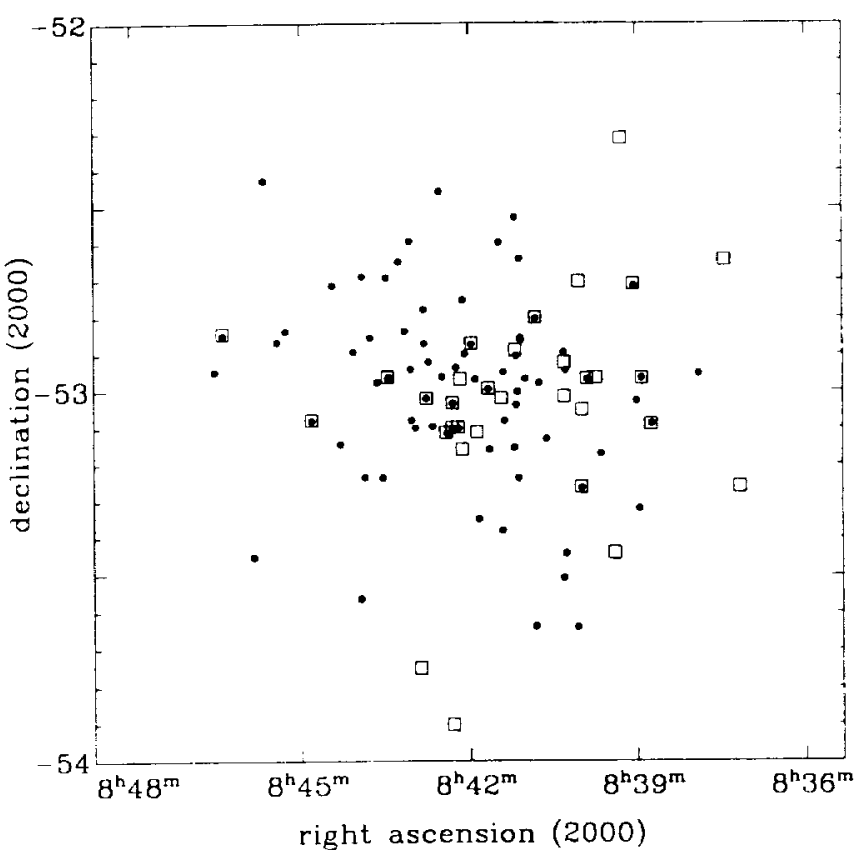

FiG. 2.-Sky map of IC 2391 showing the positions of cluster members (open squares) and X-ray sources detected by the ROSAT PSPC (filled circles).

Net X-ray counts for these 19 sources were extracted from the image using the aperture photometry routines in PROS. None of these sources showed strong evidence of variability greater than a factor of 2 in their X-ray light curves. These net counts were then transformed into count rates using the effective integration time determined at each source position on the exposure map, to correct for vignetting and obscuration by the PSPC window support structure. The conversion factor from count rate to unabsorbed flux in the ROSAT bandpass $(0.2-2.0$
keV) was determined by fitting simple, two-component. Raymond-Smith spectra to the brightest unobscured sources in the center of the image. Because the reddening to IC 2391 is extremely small, $E_{B-v}=0.004 \pm 0.003$ (Hogg 1960), we assumed $\log N\left(\mathrm{H}_{\mathrm{I}}\right)=19.4 \mathrm{~cm}^{-2}$ for this calculation. We settled on a conversion factor of $7.1 \times 10^{-12}{\mathrm{ergs} \mathrm{cm}^{-2} \mathrm{~s}}^{1}$ per PSPC count $s^{-1}$, which is consistent with other determinations in the literature (e.g., Stern et al. 1992; Fleming et al. 1992). We note that this conversion factor does have a color dependence which is not taken into account here. Finally. assuming a common distance of $162 \mathrm{pc}$ for all members of IC 2391 , we computed the X-ray luminosity for each star. Fourteen stars in our survey appear to have no X-ray counterparts. and for these we estimated $3 \sigma$ upper limits for $L_{\mathrm{x}}$ from the photon statistics following the Bayesian approach of Kraft, Burrows, \& Nousek (1991). The complete results are presented in Table 1.

\section{DISCUSSION}

We present in Figure 3 a plot of $X$-ray luminosity versus $B-V$ color for the currently known IC 2391 members. For comparison, data for the Hyades from the ROSAT All Sky Survey (Stern et al. 1992) and PSPC pointings (Stern et al. 1992, 1993; Simon \& Drake 1993) are also shown. At $B-V<0.4$, few high-mass stars remain on the main sequence in the Hyades, and so no real comparison with IC 2391 is possible. The most striking aspect of this comparison is that the overall spread of the X-ray luminosities in IC 2391 is not greatly dissimilar from that of the much older Hyades.

\subsection{The High-Mass Stars}

Our survey list contains seven stars of spectral type B, four of which have $\mathrm{X}$-ray detections. The three strongest of these sources are the late-B stars HD 74071, HD 73952, and HD 74535. The only early B star detected, HD 74560, has a lumi-

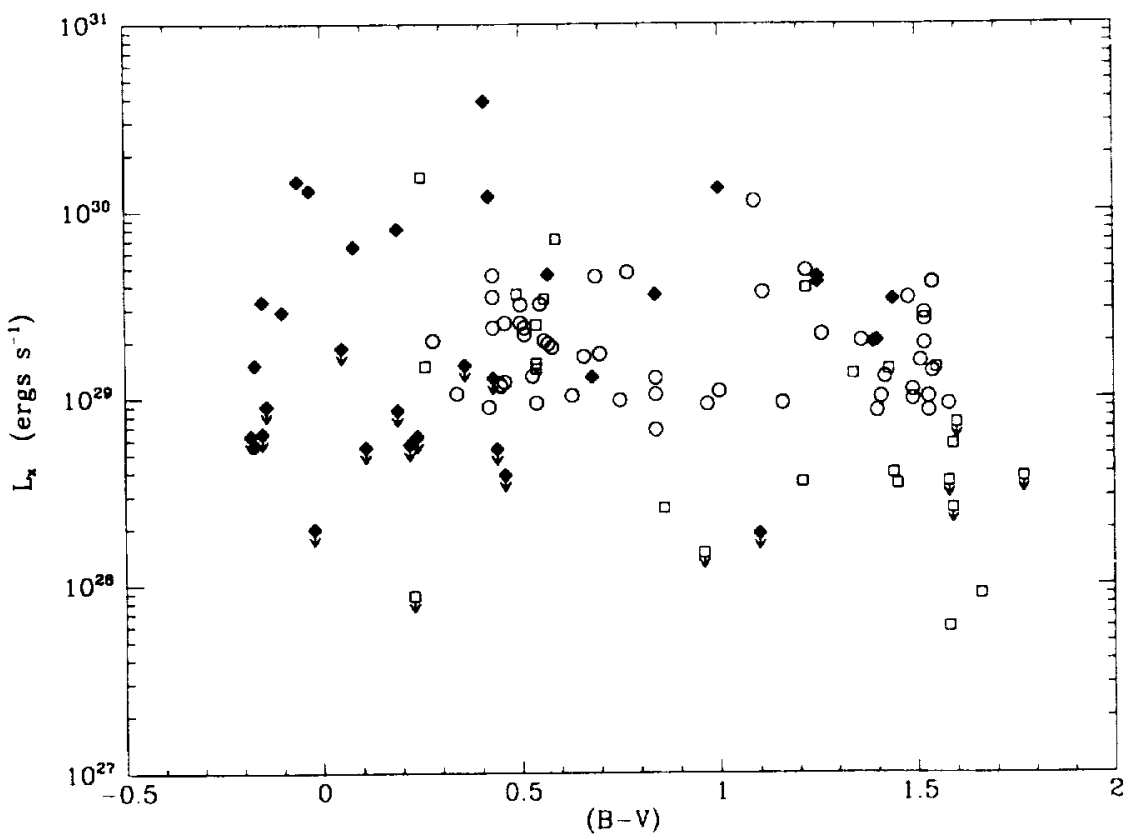

FIG 3-Plot of X-ray luminosity vs. $B-V$ for members of IC 2391 and the Hyades. Open circles represent All Sky Survey data for the Hyades (Stern et ai. 1992 ).

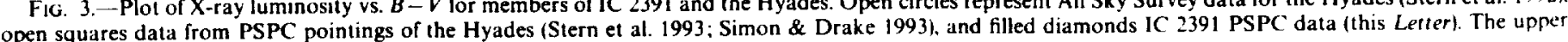
limits shown are $3 \sigma$ values. 
TABLE 1

PSPC RESUlts FOR KNOWN MEMBERS OF IC 2391

\begin{tabular}{|c|c|c|c|c|c|c|c|}
\hline Name & $\begin{array}{c}\text { Spectral } \\
\text { Type }\end{array}$ & $V$ & $B-V$ & $\begin{array}{c}\text { PSPC } \\
\text { Exposure }^{b} \\
\text { (s) }\end{array}$ & $\begin{array}{c}\text { X-ray } \\
\text { Counts }\end{array}$ & $\log L_{\mathrm{x}}^{d}$ & Notes \\
\hline HD 74195 & $\mathrm{~B} 3 / \mathrm{S} \vee(\mathrm{SB})$ & 3.64 & -0.18 & 19758 & $<57$ & $<28.80$ & \\
\hline HD $74560 \ldots \ldots \ldots \ldots$ & B3 IV (SB) & 4.89 & -0.17 & 22349 & 57 & 28.75 & \\
\hline HD $74535 \ldots \ldots \ldots \ldots$ & B9.5 III & 5.56 & -0.17 & 22448 & 154 & 29.18 & $1,2,3$ \\
\hline HD $74146 \ldots$ & B5 IV (SB) & 5.21 & -0.15 & 17891 & $<52$ & $<28.81$ & \\
\hline HD $74071 \ldots \ldots \ldots \ldots \ldots$ & B6 $\mathrm{V}$ & 5.50 & -0.15 & 12621 & 188 & 29.52 & 4 \\
\hline HD $74196 \ldots \ldots \ldots \ldots \ldots$ & $\mathrm{B} 9 / \mathrm{A0}$ (SB) & 5.60 & -0.14 & 20722 & $<86$ & $<28.96$ & \\
\hline HD $73952 \ldots \ldots \ldots \ldots \ldots$ & B8 V & 6.47 & -0.10 & 15580 & 203 & 29.46 & \\
\hline HD $74169 \ldots \ldots \ldots \ldots$ & A2p (SB) & 7.27 & -0.06 & 14088 & 930 & 30.16 & 1,4 \\
\hline HD $74275 \ldots \ldots \ldots \ldots \ldots$ & $\mathrm{A0} \mathrm{Vb}(\mathrm{SB})$ & 7.31 & -0.03 & 17653 & 1040 & 30.11 & $i$ \\
\hline HD $74516 \ldots \ldots \ldots \ldots$ & Al $\mathrm{V}$ & 7.41 & -0.02 & 21891 & $<20$ & $<28.30$ & 1 \\
\hline HD $73681 \ldots \ldots \ldots \ldots$ & Al V & 7.89 & 0.05 & 13766 & $<118$ & $<29.27$ & 1 \\
\hline HD $74955 \ldots \ldots \ldots \ldots$ & $A 1 V(S B)$ & 7.60 & 0.08 & 15868 & 459 & 29.80 & \\
\hline HD $74517 \ldots \ldots \ldots \ldots \ldots$ & $A 3 \vee(S B)$ & 8.63 & 0.11 & 22186 & $<55$ & $<28.74$ & 1,5 \\
\hline HD $75202 \ldots \ldots \ldots \ldots \ldots$ & A3 IV (SB?) & 7.75 & 0.19 & 14078 & 528 & 29.92 & \\
\hline HD $74665 \ldots \ldots \ldots \ldots$ & $A 3 \mathrm{Vn}(\mathrm{SB})$ & 8.17 & 0.19 & 13721 & $<55$ & $<28.94$ & \\
\hline HD $74044 \ldots \ldots \ldots \ldots$ & $\mathrm{A} 3 \mathrm{~m}$ & 8.53 & 0.22 & 12130 & $<32$ & $<28.76$ & \\
\hline HD $74145 \ldots \ldots \ldots \ldots$ & $\mathrm{A} 7 / 8 \mathrm{III} / \mathrm{IVm}$ & 8.57 & 0.24 & 13180 & $<38$ & $<28.80$ & \\
\hline HD $74117 \ldots \ldots \ldots \ldots$ & $\mathbf{F} 2 \mathrm{IV} / \mathrm{V}$ & 9.10 & 0.36 & 8349 & $<57$ & $<29.18$ & \\
\hline HD $74009 \ldots \ldots$ & F3 V & 8.78 & 0.41 & 15838 & 2787 & 30.59 & \\
\hline CPD $-52^{\circ} 1602 \ldots \ldots \ldots \ldots$ & F6 V & 9.69 & 0.42 & 22448 & 1234 & 30.08 & $1,3,4$ \\
\hline HD $73722 \ldots \ldots \ldots \ldots \ldots$ & F5 V & 8.92 & 0.43 & 12959 & $<76$ & $<29.11$ & 2,6 \\
\hline HD $74561 \ldots \ldots \ldots \ldots \ldots$ & F3 V & 9.38 & 0.44 & 7781 & $<19$ & $<28.73$ & 6,7 \\
\hline HD $74340 \ldots \ldots \ldots \ldots \ldots$ & F6 V & 9.88 & 0.46 & 21141 & $<37$ & $<28.59$ & 5 \\
\hline SHJM $2 \ldots \ldots \ldots \ldots \ldots$ & $\ldots$ & 10.34 & 0.57 & 21914 & 451 & 29.66 & \\
\hline SHJM $\backslash \ldots \ldots \ldots \ldots \ldots$ & $\ldots$ & 10.29 & 0.68 & 15973 & 95 & 29.12 & \\
\hline SHJM $6 \ldots \ldots \ldots \ldots \ldots$ & $\ldots$ & 11.86 & 0.84 & 12883 & 213 & 29.56 & \\
\hline SHJM $3 \ldots \ldots \ldots \ldots$ & $\ldots$ & 12.63 & 1.00 & 15807 & 956 & 30.12 & \\
\hline SHJM $7 \ldots \ldots$ & $\ldots$ & 12.52 & 1.10 & 22341 & $<20$ & $<28.29$ & \\
\hline SHJM $8 \ldots \ldots \ldots \ldots \ldots$ & $\ldots$ & 13.38 & 1.25 & 22462 & 450 & 29.64 & 8 \\
\hline SHJM $9 \ldots \ldots \ldots \ldots$ & $\ldots$ & 13.58 & 1.25 & 21314 & 404 & 29.62 & 8 \\
\hline SHJM $4 \ldots \ldots \ldots \ldots \ldots$ & $\ldots$ & 14.21 & 1.39 & 21010 & 194 & 29.31 & 9 \\
\hline SHJM $5 \ldots \ldots \ldots \ldots \ldots$ & $\ldots$ & 14.21 & 1.39 & .21010 & 194 & 29.31 & 9 \\
\hline SHJM $10 \ldots \ldots \ldots \ldots \ldots$ & $\ldots$ & 14.00 & 1.44 & 22340 & 345 & 29.53 & \\
\hline
\end{tabular}

Nores-Unless otherwise noted, photometry is from Perry \& Hill 1969 and spectral type is from Houk 1978 (1) Photometry from Hogg 1960 ; (2) possible nonmember; (3) CPD-52 1602 and HD 74535 X-ray sources blended. Estimated flux ratio is $8: 1$ respectively; (4) spectral type from Levato \& Malaroda 1984; (5) spectral type from Buscombe $1965 ;(6) B-V$ from SIMBAD; 7 spectral type and $V$-magnitude from Levato \& Malaroda 1984; (8) these two stars only weakly detected at the chromospheric $\mathbf{M g}$ il lines (Simon 1990). Their X-ray emission appears unusually bright, given their UV emission and rotation rates; (9) SHJM 4 and 5 are separated by $3^{\prime \prime}$. The X-ray flux is divided evenly between these two stars.

- SB = spectroscopic binary

- Effective exposure time, in seconds, as measured from the exposure map at the position of the source.

c Net source counts, corrected for background, measured using the PROS software. The gross counts were measured within a circular aperture of $120^{\prime \prime}$ radius ( $180^{\prime \prime}$ for far off-axis sources), background counts within concentric annuli of radii $150^{\prime \prime}$ and $250^{\prime \prime}$ (195" and $300^{\prime \prime}$ for far off-axis sources).

${ }^{d} \log L_{x}$, corrected for interstellar extinction, in units of ergs s ${ }^{-1}$. We assume $d=162 \mathrm{pc}$.

nosity of $5.6 \times 10^{28} \mathrm{ergs} \mathrm{s}^{-1}$, which is near our detection limit. HD 74071 is the only B star detected whose X-ray luminosity is consistent with the canonical relation $L_{\mathrm{x}} / L_{\text {bol }} \approx 10^{-7}$ for $\mathrm{O}$ and B stars (Long \& White 1980; Pallavicini et al. 1981). For the remaining three detections, HD 74560 is $\sim 20$ times underluminous, HD 73952 is $\sim 4$ times overluminous, and HD 74535 is $\sim 5$ times overluminous with respect to this relation. We were especially surprised that $o \mathrm{Vel}, \mathrm{B} 3 / 5 \mathrm{~V}$, the visually brightest cluster member, was only seen as an upper limit in our image, and is thus more than 10 times underluminous. It is thought that the X-ray emission of early B-type stars is produced by shocks in radiatively driven winds (MacFarlane \& Cassinelli 1989). However, the mechanism involved in producing those shocks is still poorly understood (MacFarlane et al. 1991). We suspect that variations in mass-loss rate and wind geometry from one star to the next may account for the wide range of X-ray emission levels exhibited by the B stars in IC
2391. Our data are consistent with the more general results of Grillo et al. (1992), whose Einstein survey of 1545 B stars showed that (a) most B stars have $L_{\mathrm{x}}<10^{29-30} \mathrm{ergs} \mathrm{s}^{-1}$, and (b) those stars detected by Einstein span a range in $L_{x}$ of almost four orders of magnitude.

Of the $10 \mathrm{~A}$ stars in our survey, the four that we detected are all classified as spectroscopic binaries. The high levels of X-ray emission seen from these four stars are likely due to late-type companions rather than the A stars themselves. The other six A stars in our survey were not seen in our image and were assigned upper limits. Because the limiting $X$-ray luminosity at the center of our image is $\approx 2 \times 10^{28} \mathrm{ergs} \mathrm{s}^{-1}$, we do not have the sensitivity necessary to test the conclusions of Schmitt et al. (1985) and Simon \& Drake (1993) that single A stars as a class are characterized by very low $\mathrm{X}$-ray emission $\left(L_{\mathrm{x}}<10^{28}\right.$ ergs $\left.\mathbf{s}^{-1}\right)$. Our upper limits are consistent with their conclusions, however. 


\subsection{The Low-Mass Stars}

For the lower main sequence of IC $2391(B-V \geq 0.4)$, we note some features in Figure 3 that are at odds with the conventional picture of stellar evolution on the main sequence painted by activity-age scaling laws, such as the Skumanich (1972) inverse square-root law. First, among the IC $2391 \mathrm{G}$ stars there are stars with $L_{\mathrm{x}}$ as weak as that observed for similar mass stars in the Hyades. Second, among the IC $2391 \mathrm{~K}$ stars, the most $\mathrm{X}$-ray luminous stars are not much more active than their counterparts in the Hyades (less than a factor of 2). Third, if SHJM 7, seen only as an upper limit, is truly an IC 2391 member, then there are stars with $L_{\mathrm{x}}$ as weak as their Hyades counterparts along the entire lower main sequence of IC 2391. And finally, only among the late-F and early-G stars do we see an IC 2391 member (HD 74009) which is clearly more luminous than any Hyades member of similar mass. In this same mass range, however, more IC 2391 members are seen as weak upper limits than as actual X-ray detections, implying there is a large spread of activity levels at any given mass. While the most highly active stars in both clusters may owe their X-ray activity to binarity, it is the lower envelope of the range of $L_{\mathrm{x}}$ seen in both clusters that is intriguing. At their weakest, the emission levels observed in the Hyades are similar to those of active, main-sequence stars in the field $\left(10^{28.3-29}\right.$ ergs $s^{-1}$ ). Though our sample from IC 2391 is limited by the small number of known members and the higher limiting $L_{x}$, as compared to the deeper Hyades pointings, there are four lowmass IC 2391 members (three upper limits and a detection) with $L_{\mathrm{x}}$ less than a factor of two from that of their active counterparts in the field.

If the activity-age relations thus far proposed for chromospheric emission (e.g., Skumanich 1972; Barry et al. 1987) or chromospheric/transition region emission (Simon et al. 1985) are extended to coronal $X$-rays, we would expect the $X$-ray emission levels of solar-type stars in IC 2391 to be much higher than those we have in fact observed. The age difference between the Hyades and IC 2391 is a factor of 10-25. If we apply the classic $t^{-1 / 2}$ scaling law (Skumanich 1972), the X-ray luminosities of the $G$ and $K$ stars in IC 2391 should be a factor of 3-5 times larger than those observed for similar mass stars in the Hyades. The enhancement should be even larger if higher temperature features have steeper activity-age relations (Simon et al. 1985). However, Figure 3 shows that neither the mean nor the maximum $L_{\mathrm{x}}$ values for these two clusters conform to such simple power-law formulas. Little help can be gained by assuming that we have failed to account for the brightest X-ray sources in IC 2391, since known cluster members correspond to the brightest $\mathrm{X}$-ray sources in our PSPC image. In the case of IC 2391, the data show that the existing empirical activity-age scaling laws are clearly wanting.

On the other hand, we believe the data for IC 2391 support our hypothesis that low-mass stars start out their mainsequence lives with a wide range of activity levels as well as rotation rates (Simon 1990, 1992). At an age of 30-50 Myr, the low-mass stars in IC 2391 have clearly only just arrived on the ZAMS, or at least have not resided there long enough to experience significant rotational spindown. Any age spread present in the cluster is therefore small, and the spread of X-ray luminosities seen in IC 2391 must reflect initial conditions at the ZAMS. If the decay of activity with age is, as most believe. governed by a feedback process between the loss of angular momentum from the star's surface layers due to magnetic braking, and the decreased strength of the stellar dynamo. which depends explicitly on the stellar rotation rate, then the subsequent evolution of coronal activity of these stars in IC 2391 should depend on these initial conditions. Magnetic braking should decelerate the surface layers most quickly for the most initially rapidly rotating, active stars, and most slowly for those which are already slowly rotating from the time of their arrival on the ZAMS. The implication is that no one empirical formula, such as the square-root relation, can describe the time decay of dynamo activity for each and every star.

Though our sample of low-mass stars in IC 2391 is small, many more previously unidentified cluster members are likely to be identified as optical counterparts of X-ray sources in our image. Because the brightest $X$-ray sources are already identified with known cluster members, we believe a large spread in $L_{x}$ along the entire lower main sequence in IC 2391 will be affirmed as new cluster members are identified with the fainter sources.

This research was supported in part by NASA grant NAG 5-2022 to the University of Hawaii. We wish to thank the staff of the ROSAT observatory, the US ROSAT Science Data Center, and the Harvard-Smithsonian Center for Astrophysics for their assistance in the acquisition and reduction of the $X$-ray data presented here. We would also like to thank Jeff Linsky for several helpful comments on the manuscript.
Barry, D. C. Cromwell, R. H. \& Hege, E. K. 1987, ApJ, 315, 264

Buscombe, W. 1965, MNRAS, 129,411

Caillaut, J.-P., Gagne, M., \& Stauffer, J. 1992, in 7th Cambridge Workshop on Cool Stars, Stellar Systems, and the Sun, ed. M. S. Giampapa \& J. A. Book binder (ASP Conf. Ser. 26), 97

Fleming T. A. Giampapa, M. S., Schmitt, J., \& Bookbinder, J. A. 1992, in 7th Cambridge Workshop on Cool Stars, Stellar Systems, and the Sun, ed. M. S. Giampapa \& J. A. Bookbinder (ASP Conf. Ser. 26), 93

Grillo, F., Sciortino, S., Micela, G., Vaiana, G. S., \& Harnden, F. R., Jr. 1992, ApIS, 81, 795

Hoge A. R. 1960, PASP, 72, 85

Houk, N. 1978, Michigan Catalog of 2-Dimensional Spectral Types for the Houk, N. 1978, Michigan Catalog of 2-Dimensional Spectral Types for the
HD Stars, Vol. 2, Zones $-52^{\circ}$ to $-40^{\circ}$ (Ann Arbor: Department of Astronomy, Univ. Michigan)

Kraft, R. P. Burrows, D. N \& Nousek, J. A. 1991, ApJ, 374, 344

Levato, H." \& Garcia, B. 1984, Ap. Lett., 24, 49

Levato, H., \& Garcia, B. 1984, Ap. \& Morrell, N. 1988, Ap\&SS, 146, 361

Levato, H., \& Malaroda, S. 1984, Ap. Lett.. 24, 37

Leva K S, \& White, R. L. 1980, ApJ, 239, L65

MacFarlane, J. J., \& Cassinelli. J. P. 1989, ApJ, 347, 1090

\section{REFERENCES}

MacFarlane, J. J., Cassinelli, J. P., Welsh, B. Y., Vedder, P. W., Vallerga. J. V.. \& Waldron, W. L. 1991, ApJ, 380,564

Pallavicini, R. Golub, L. Rosner, R., Vaiana, G. S., Ayres, T., \& Linsky, J. L. 1981, ApJ, 248, 279

Perry, C. L., \& Bond, H. E. 1969, PASP, 81.629

Perry, C. L. \& Hill, G. 1969, AJ, 74, 899

Schmitt, J. H. M. M., Golub. L., Harnden, F. R., Jr., Maxson, C. W., Rosner. R.

\& Vaiana, G.S. 1985, ApJ, 290, 307

Simon, T. 1990, ApJ, 359, L51

1992, in 7 th Cambridge Workshop on Cool Stars, Stellar Systems. and the Sun, ed. M. S. Giampapa \& J. A. Bookbinder (ASP Conf. Ser. 26), 3

Simon, T., \& Drake, S. 1993. AJ, submitted

Simon, T Herbio G. H \& Boesgaard, A. M. 1985, ApJ, 293, 551

Skumanich, A. 1972, ApJ, 171, 565

Stauffer, J., Hartmann, L. W., Jones, B. F., \& McNamara, B. R. 1989, ApJ. 342. 285

Stern, R. A. Schmitt, J. H. M. M.. Pye, J, P., Hodgkin, S. T.. Stauffer, J. R.. \& Simon, T. 1993, ApJ, submitted

Stern, R. A., Schmitt, J. H. M. M., Rosso, C., Pye, J. P., Hodgkin. S. T.. \& Stauffer, J. R. 1992, ApJ, 399, L159 


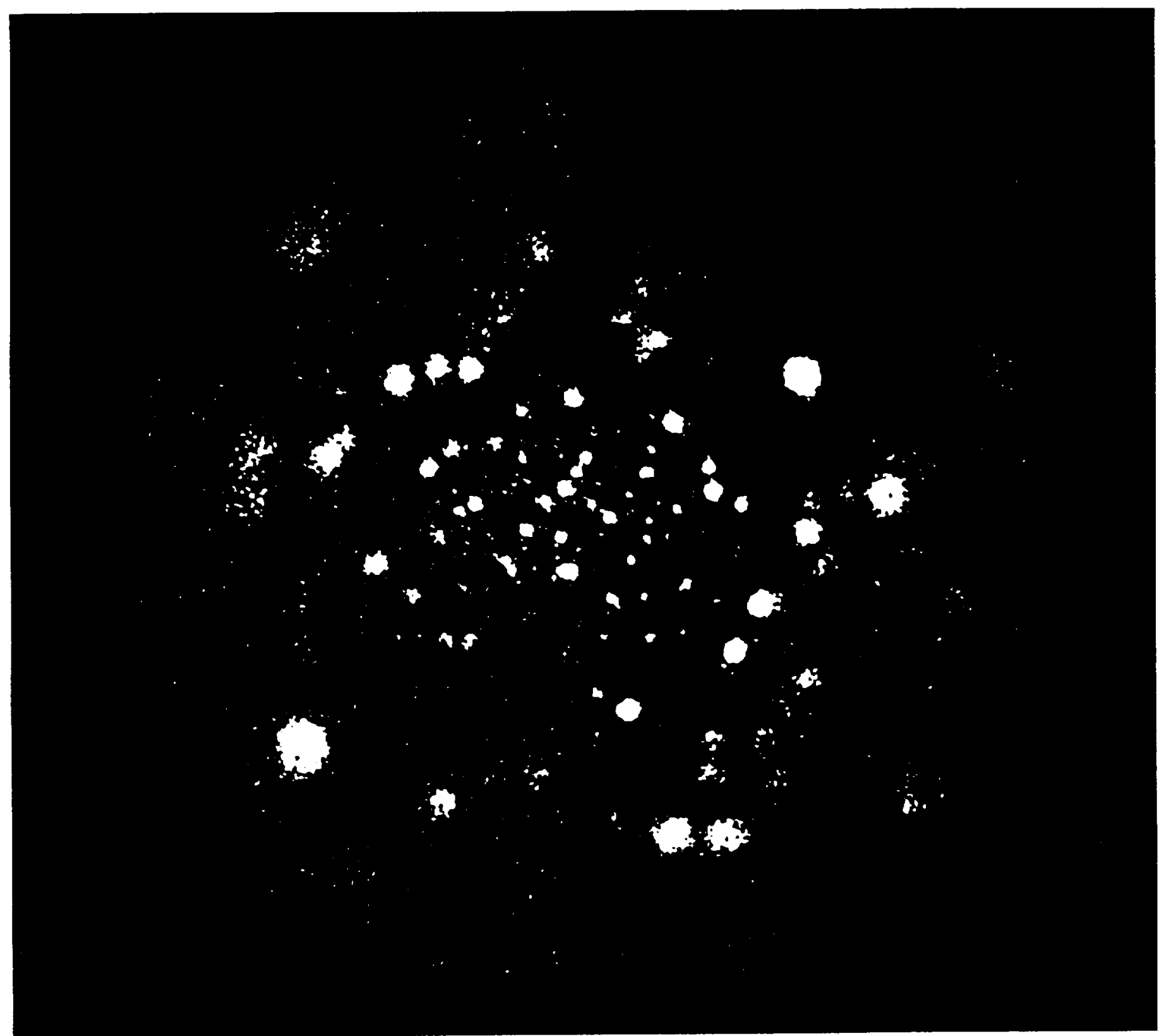

Fic . 1.-A $23 \mathrm{ksec}$ ROSAT PSPC observation of the young cluster IC 2391. This false color image is centered at R.A. $=8^{\mathrm{h}} 42^{\mathrm{m}} 00^{\mathrm{s}}$, Decl. $=-5300^{\prime} 36^{\prime \prime}$ and has a lield of view of 2 . The original image has been rebinned to $5^{\prime \prime}$ pixels and then smoothed by a Gaussian of 23 ". 5 FWHM, in order to reduce the background noise. There are 76 sources present in this image with $L_{x} \geq 2 \times 10^{28} \mathrm{ergs} \mathrm{s}^{-1}$.

Patten \& Simon isee 415, Li23) 
